\title{
EXPERIMENTAL INVESTIGATION OF THIN BRASS SHEETS UNDER TENSION-COMPRESSION CYCLIC LOADING
}

\author{
Zbigniew L. Kowalewski, Lech Dietrich \\ Institute of Fundamental Technological Research, Polish Academy of Sciences, Warsaw, Poland \\ e-mail: zkowalew@ippt.pan.pl \\ Grzegorz Socha \\ Institute of Aviation, Warsaw, Poland
}

\begin{abstract}
Strength and durability of thin-walled structures are usually calculated with the use of computer simulations. To perform such simulations using Finite Element Method, characteristics of a material subjected to monotonic tension or compression and tension-compression cyclic loading are necessary. Experimental determination of such kind of characteristics is usually performed on specimens cut out from a metal or composite thin sheet. Problems associated with testing on flat specimens under large deformation are discussed in this paper. A new design of fixture proposed by the authors for this kind of testing is shortly described. The results of investigations carried out on brass using the new fixture for flat specimens testing are also presented.
\end{abstract}

Keywords: fixture, large deformations, tension-compression tests, thin metal sheet

\section{Introduction}

Problems associated with material testing on flat specimens under compression within a large deformation range procure many difficulties. It seems that buckling is regarded as the most significant. Many propositions of experimental setups enabling compression testing of flat specimens were given by researchers (see References). In 1978, Dietrich and Turski (1978) elaborated solution of the side-supporting fixture. The main advantage of this design was the ability to support the entire specimen gage length during a test. This was due to the fact that the sidesupporting block was able to change its length together with gradual shortening of the specimen during compression. A detailed description of the fixture was given in the monograph published by Szczepiński (1990).

The fixture is shown in Fig. 1a. The main principle of operation is explained in Fig. 1b where two expanded views of parts of the fixture are shown. The maximum and minimum height of the compression fixture is given in Fig. 1a. The side-support of the specimen has been realized by two sets of thin plates perpendicular to the specimen surface supporting its both sides. Each set, consisting the same type of the plate is positioned by two pins: the first is inserted into the pin-hole at one end of the plate and the second is inserted into U-shape cut on the opposite side of the plate. The neighboring plates are rotated with respect to each other by an angle equal to $180 \mathrm{deg}$, see Fig. 1b. The upper and lower yoke support both pins. The screws connecting two parts of the yoke allow the adjustment of the fixture to fit the specimen thickness.

In this paper, a modified version of the fixture developed in the 70's (Dietrich and Turski, 1978) is applied to execute experimental investigations of thin metal sheets under tensioncompression cyclic loading. It enables application of cyclic tension-compression to the flat specimen in a wide strain range due to coupling of the side-supporting blocks with the standard grips of the testing machine. The main principle of the fixture assembly is explained in Fig. 2, 
(a)

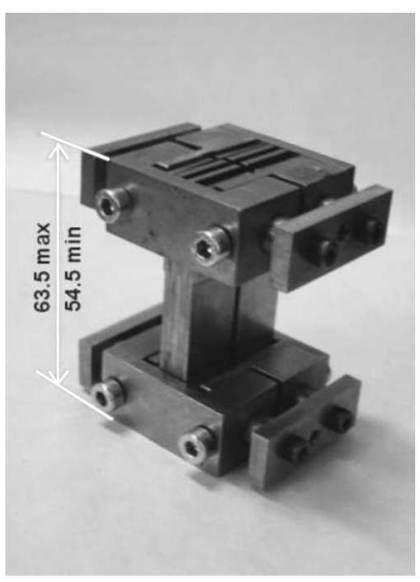

(b)

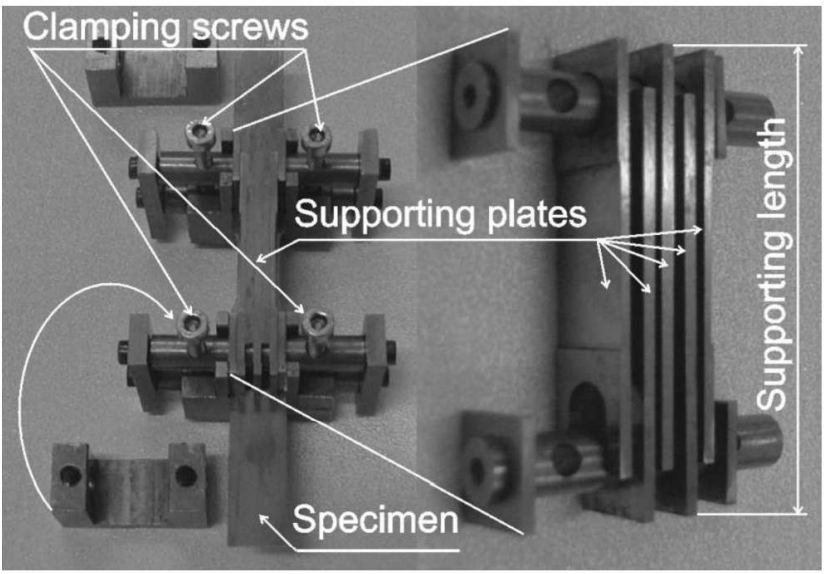

Fig. 1. (a) A special fixture for compressive tests. (b) Expanded views of supporting plates and the fixture with mounted specimen and four clamping bolts

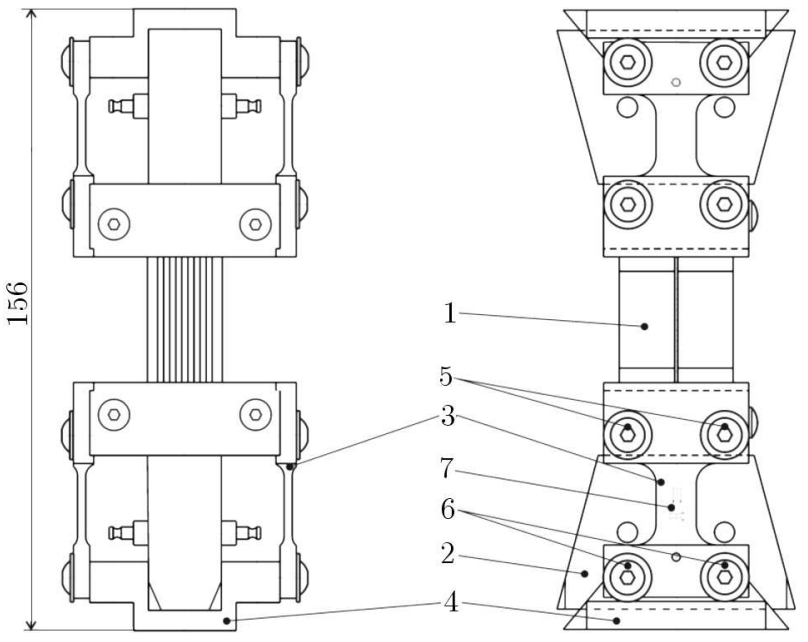

Fig. 2. Technical drawing of the fixture and numbered component parts: front and side view

where engineering drawing of the fixture is presented. The main part of the fixture placed in the middle (1) is exactly the same design of the side-supporting block as that developed in 1978. The gripped part of the specimen is held by wedges (2) comprising the standard grip assembly of the testing machine and operated thanks to hydraulic pressure supplying that machine. The complete assembly of the sliding block is fixed to the testing machine using screws $(5,6)$, connectors (3) and base (4). More details of this device can be found in (Dietrich et al., 2014).

The design from the 70's could have been used only for monotonic compression since supporting blocks once shortened remained in this position. Another important advantage of the modified design is the ability of monitoring the friction force between the specimen and supporting blocks, which allows avoiding the error during stress determination. The measurement of the friction force is realized by strain gages (7) cemented to connector (3).

\section{Experimental details}

\subsection{Specimen and material}

All tension-compression tests have been carried out on thin sheet specimens with nominal thickness equal to $1 \mathrm{~mm}$ using the fixture that is briefly presented in the previous Section. Dimensions of the specimen are shown in Fig. 3. 


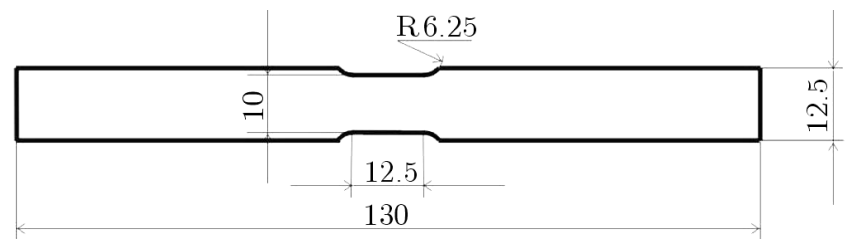

Fig. 3. The specimen manufactured from a $1 \mathrm{~mm}$ thickness sheet of brass

The material tested has been M63 brass used in deep-drawing processes. The chemical composition of the alloy is presented in Table 1 according to Polish Standards.

Table 1. Chemical composition of M63 brass (CW508L notation according to EN)

\begin{tabular}{|c|c|c|c|c|}
\hline $\mathrm{Cu}$ & $\mathrm{Ni}$ & $\mathrm{Fe}$ & $\mathrm{Pb}$ & $\mathrm{Zn}$ \\
\hline \hline $62-64$ & $\max 0.30$ & $\max 0.10$ & $\max 0.10$ & rest \\
\hline
\end{tabular}

\subsection{Preliminary tensile tests}

Before tension-compression cycles, the standard tensile test has been carried out in order to determine mechanical properties of the brass tested. It has been performed without application of the anti-buckling fixture. Instead of it, typical grips and mechanical extensometer have been used. The determined mechanical parameters are shown in Table 2. The results of tensile test are also used to validate the experimental data from tension-compression cycles.

Table 2. Mechanical parameters of M63 brass

\begin{tabular}{|c|c|c|c|}
\hline Young's modulus & Yield point & Tensile strength & Total elongation \\
\hline \hline $110 \mathrm{GPa}$ & $550 \mathrm{MPa}$ & $680 \mathrm{MPa}$ & $14 \%$ \\
\hline
\end{tabular}

\subsection{Experimental program}

The cyclic loading has been carried out under displacement control with the rate $0.05 \mathrm{~mm} / \mathrm{s}$. The conditions on the engineering strain have been set to limit the strain range during cycling.

In the first type of tests (tension-compression), 10 cycles within a strain range \pm 0.040 (strain varied between 0.02 and -0.02 ) have been executed starting in the tensile direction. The last cycle ended with force equal to zero.

In the second type of tests, a similar program has been realized in the compressive range of strain changing from -0.002 to -0.020 . As in the former case, 10 cycles have been executed ending with the zero force.

All tests have been carried out using an extensometer with the range of \pm 0.2 . The load cell has been calibrated in the range of $\pm 25 \mathrm{kN}$. The special set-up for friction force measurement has been applied. It consisted of two coupling bars with strain gauges calibrated in the range of $\pm 2 \mathrm{kN}$.

\section{The results of cyclic tests}

The results of the first type of tests carried out on M63 brass under cyclic loading are presented in Fig. 4. The first cycle is illustrated by the solid black line denoted as 1 . The tensile stressstrain curve obtained under simple tension without using the anti-buckling fixture is also shown in Fig. 4 (gray dotted line denoted as 2). 


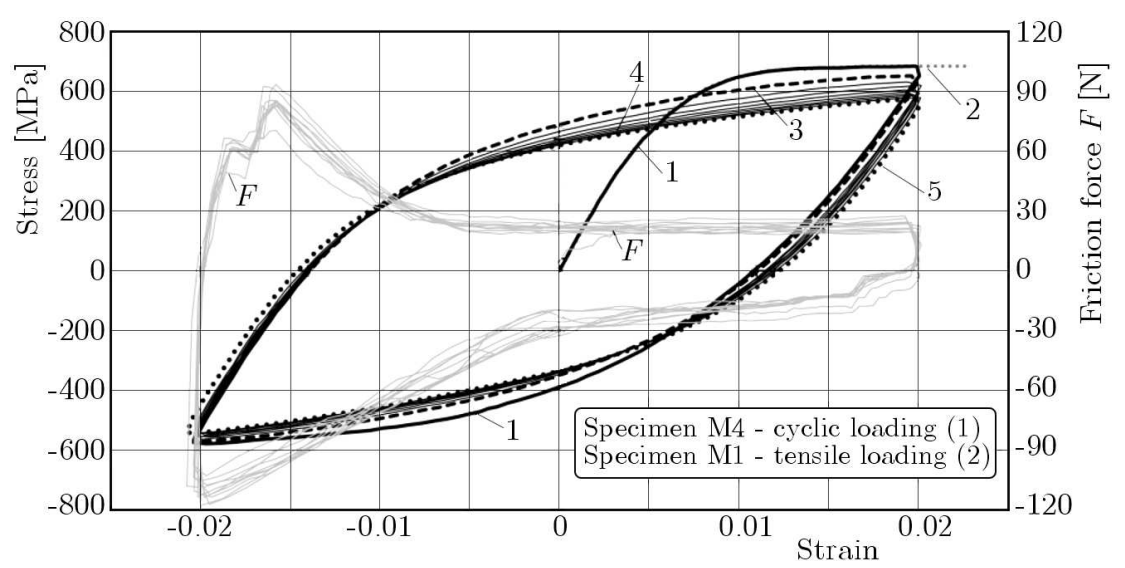

Fig. 4. Hysteresis loops of the brass and variation of the friction force during the test

The second cycle is represented by black dashed line (3). The last two cycles are denoted by black dotted lines ( 4 and 5). Figure 4 also presents the evolution of the friction force (gray lines, denoted as $F$ ). The friction force is also shown as a function of time in Fig. 5a as well as the total force for all recorded cycles. Changes of strain corresponding to the force variations are shown in Fig. 5b for all cycles carried out.
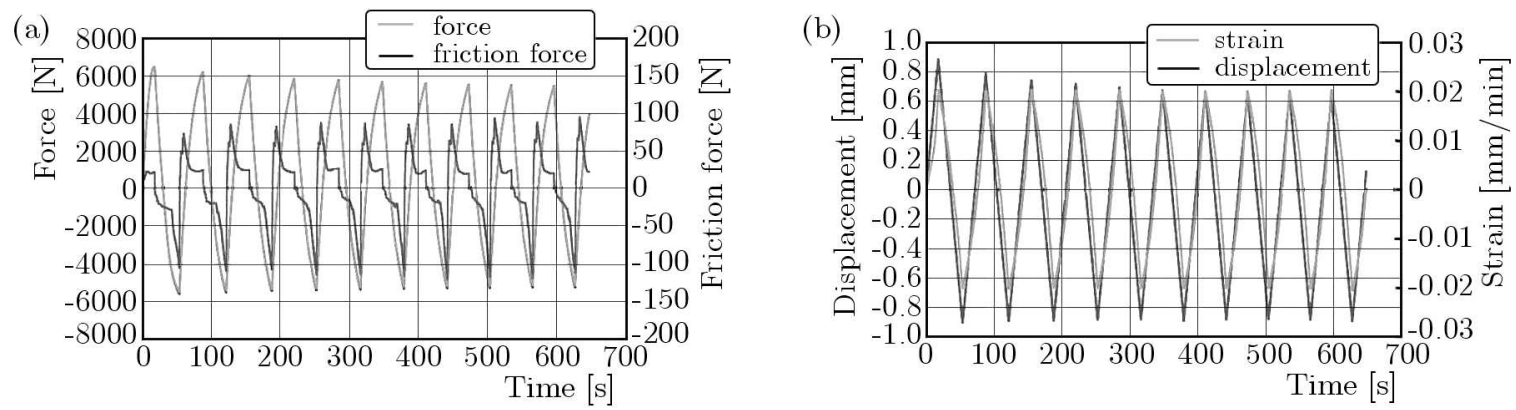

Fig. 5. Variation of the specimen load and friction force (a) and variation of displacement and strain (b) versus time during cyclic loading (type 1)

The brass exhibited the softening effect reflected by a significant decrease in the stress amplitude, especially in the first two cycles, see Fig. 5a.

The level of the friction force was also monitored during the test. Its variation is presented in Figs. 4 and 5a. The friction force has a similar course in all cycles and does not change clearly under tension and grows up at compression. It has to be emphasized, however, that values of the friction force are relatively small and they do not change the cyclic stress-strain characteristic.

In the second scheme of tests, the cyclic loading has been applied for the strain level varying between -0.002 and -0.020 . Also ten cycles have been carried out, however in that case, the loading process started in the compression direction. The results of the second type of tests are presented in Fig. 6.

The softening effect tokes place for the material in question. It is most remarkable for the first two cycles. During subsequent cycles, the saturation state is almost achieved, i.e. hysteresis loops coincide themselves. As it is clearly seen in Figs. $7 \mathrm{a}$ and $7 \mathrm{~b}$, the friction force has rather low magnitudes. As in the previous cases, the friction force variation data are subsequently used to correct the stress-strain characteristic of the brass tested. 




Fig. 6. Hysteresis loops of the brass and variation of the friction force during the test
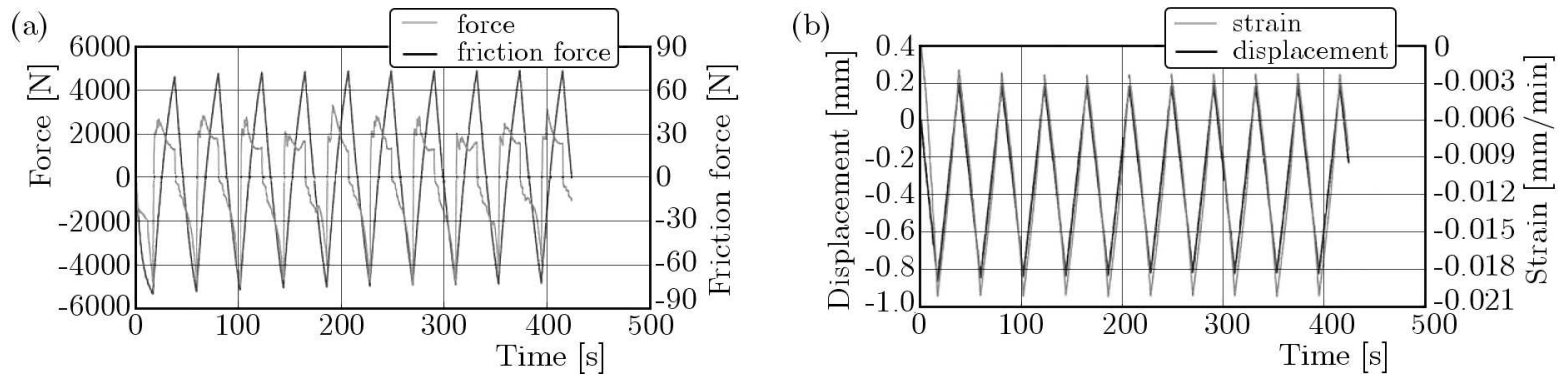

Fig. 7. Variation of the specimen load and friction force (a) and variation of displacement and strain (b) versus time during cyclic loading (type 2)

\section{Concluding remarks}

Taking into account all data captured by means of the new fixture, one can conclude that the technique is promising with respect to providing data for the modeling of cyclic deformation behavior for shell structures. It enables one to avoid buckling during compression of specimens made of thin metal sheets. The fixture changes its length with specimen elongation or shrinkage during a test which allows application of the cyclic load.

Although in the case of the presented tests the strain range has been set to \pm 0.02 , the fixture nominal strain capacity is much greater. Depending on mutual location of the supporting plates, the testing technique allows tension-compression tests to be performed at the displacement amplitude within the range $\pm 5 \mathrm{~mm}$ what corresponds to the maximum strain amplitude of \pm 0.4 for the specimen gage length to be equal $12.5 \mathrm{~mm}$.

The friction force, which is generated due to movement of both parts of the fixture, is measured by the special strain gauge system during each test. It allows essential reduction of the friction force influence on the stress-strain characteristics.

\section{References}

1. Aitchison C.S., Tuckerman L.B., 1939, The "pack" method for compressive tests of thin specimens of materials used in thin-wall structures, NACA, 649

2. Cao J., Lee W., Cheng H. S., Seniw M., Wang H. P., Czung K., 2009, Experimental and numerical investigation of combined isotropic-kinematic hardening behavior of sheet metals, International Journal of Plasticity, 25, 942-972 
3. Dietrich L., Socha G., Kowalewski Z.L., 2014, Anti-buckling fixture for large deformation tension-compression cyclic loading of thin metal sheets, Strain International Journal of Experimental Mechanics, 50, 174-183

4. Dietrich L., Turski K., 1978, A new method of thin sheets testing under compression (in Polish), Engineering Transactions, 26, 1, 91-99

5. JACKMAN K. R., 1944, Improved methods for determining the compression properties of sheet metal, Automotive and Aviation Industries, 90, 11, 36

6. Kuwabara T., 2007, Advances in experiments on metal sheets and tubes in support of constitutive modeling and forming simulations, International Journal of Plasticity, 23, 385-419

7. Kuwabara T., Kumanto Y., Ziegelheim J., Kurasaki I., 2009, Tension-compression asymmetry of phosphor bronze for electric parts and its effect on bending behavior, International Journal of Plasticity, 25, 1759-1776

8. LaTour H., Wolford S., 1945, Single-strip compression test for sheet materials, Proc. ASTM, 45, 675

9. Miller J. A., 1946, A fixture for compressive tests of thin sheet metal between lubricated steel guides, NACA, 1022

10. Sandorff P.E., Dillon R.K., 1946, Compressive stress-strain properties of some aircraft materials, Proc. ASTM, 46, 1039

11. Standard methods of compression testing of metallic materials, ASTM E9-61

12. Szczepiński W., EDIT., 1990, Experimental Methods in Mechanics of Solids, PWN, Warszawa, Elsevier, Amsterdam-Oxford-New York-Tokyo

Manuscript received March 2, 2015; accepted for print April 27, 2015 